\title{
An ultra-wideband Rotman lens using modified dummy sidewalls
}

\author{
Moein Nazari ${ }^{\mathrm{a})}$, Ahmad Mortezaie, Ayaz Ghorbani, \\ and Gholamreza Moradi \\ Amirkabir University of Technology \\ No.424, Hafez Ave, Tehran, Iran \\ a)moein_nazari@aut.ac.ir
}

Abstract: A wideband design model for $4 \times 4$ microstrip Rotman lens is presented, with a precise behavior of return loss of the ports and their phase centers. The procedure for choosing the optimum geometrical parameters of lens and modifying the curvature of sidewalls yield a very wideband lens. Based on the presented model, a prototype has been built for the true time delay phasing of an antenna array for the frequency range from $8 \mathrm{GHz}$ to $26 \mathrm{GHz}$. The fabricated $4 \times 4$ Rotman lens has an average amplitude error of $0.5 \mathrm{~dB}$ and phase errors of 2 degree. All the beams have a fixed position in frequency band and the maximum deviation of the beams from the ideal position is less than 0.65 degree.

Keywords: Rotman lens, true-time delay, beamforming

Classification: Microwave and millimeter wave devices, circuits, and systems

\section{References}

[1] W. Rotman and R. Turner, "Wide-angle microwave lens for line source applications," IEEE Trans. Antennas Propag., vol. AP-11, no. 6, pp. 623632, Nov. 1963.

[2] T. Katagi, "An improved design method of rotman lens antennas," IEEE Trans. Antennas Propag., pp. 524-527, 1984.

[3] P. S. Hall and S. J. Vetterlein, "Reviewof radio frequency beamforming techniques for scanned and multiple beam antennas," Inst. Elect. Eng. Proc. H, Microwaves Antennas Propag., vol. 137, no. 5, pp. 293-303, Oct. 1990.

[4] D. H. Archer and M. J. Maybell, "Rotman Lens Development History at Raytheon Electronic Warfare Systems 1969-1995," Proc. IEEE Antennas Propag. Symp., vol. 2B, 3-8, pp. 31-34, July 2005.

[5] R. C. Hansen, "Design trades for Rotman lenses," IEEE Trans. Antennas Propag., vol. 39, no. 4, pp. 464-472, April 1991.

[6] J. Dong, "Microwave Lens Designs: Optimization, Fast Simulation Algorithms, and 360-degree Scanning Techniques," Sept. 2009.

[7] L. T. Hall, "Broadband Monolithic Constrained Lens Design," The University of Adelaide, Aug. 2009. 


\section{Introduction}

A Rotman lens is a parallel plate structure used as beamforming network for a linear array of antennas [1]. The advantage of Rotman lens includes monolithic construction, low profile and low cost, light weight, ease of construction and simultaneously availability of many beams. But, the most important feature of the lens is its capability to produce fixed beams in extremely wideband operation. These features make the Rotman lens an attractive candidate for use in multibeam purposes $[2,3]$.

The Rotman lens is a useful, true time delay beamformer, which has been fully studied by geometrical optics (GO) over the past decades. The lens equations for a three-focii waveguide lens were derived in [1], converted for a microstrip lens in [4]. The geometrical parameters of lens is derived from GO. The GO approximations are often not reliable enough for wideband microstrip lens design. Major source of inaccuracies are reflections off sidewall of dummy ports, coupling between the microstrip feed lines and errors in assuming the position of the phase center of the feed taper. The GO approximation cannot consider these problems. Therefore; the sidewall contours must be design so carefully to achieve an optimal performance. It worth to be mention, there is not a definite perfect lens design. But, always there is a tradeoff between size, shape and phase errors.

Based on the three-focii lens design equations [5], the input contour and the output contour of lens can be constructed once the following parameters such as the number of beams, the number of arrays, maximum scan angle, focal ratio, off center focal angle, ray to beam angle ratio, transmission line length, center frequency, bandwidth, the eccentricity of the beam contour and the dielectric constant of substrate are decided.

The design parameters affect the shape of microwave lens, as well as the phase error performance [5].

The focal ratio parameter is considered as the optimization factor in most recent works. In $[6]$ it is found that different focal ratio $(g=1 / \beta)$ values yields different phase error. The $\mathrm{F} / \mathrm{D}$ ratio is another important factor during the phase error optimization process. Results show that by increasing the F/D ratio the phase error decreases [7].

\section{Design a three-focii Rotman lens}

The proposed Rotman lens, see Fig. 1 (a), is realized on Rogers RO4003 with a permittivity of 3.38 . The dimensions of prototype lens are $18 \mathrm{~cm} \times$ $20.5 \mathrm{~cm}$. This lens has four beam port, four array port and 22 dummy ports. The maximum scan angle is \pm 22.5 degree. For this lens design uniform amplitude and linear phase variation is desired.

Firstly, we design the Rotman lens by the GO equations found in [4]. Moreover, we also consider the optimum $\mathrm{g}=1.023$ for $\mathrm{F} / \mathrm{D}$ ratio equal to 1.4. Also, we choose the focal angle parameter $(\alpha)$ equal to maximum scan angle. In this way, two of outer beam ports placed on the focal points. Therefore; we expect that for these beam ports the phase errors are equal 
to zero. GO calculations show that the maximum phase error for all array ports is less than 0.15 degree.

\section{Side wall modification for improving the Bandwidth}

We noticed that the mutual coupling between microstrip feed lines and the multi reflections are not predictable by GO. Therefore; to draw a reasonable estimation of the amplitude and phase information, numerical methods are desired. Also, for minimizing the multiple reflections from sidewalls, the sidewall contour must be modified. The shape of the lens sidewall dictates how efficiently energy faced the wall is absorbed.

To achieve an optimal contour and proper design of dummy ports, the angle of dummy ports to incident wave must be chosen carefully. Full wave optimization shows that if dummy ports point to the middle of beam/array port contour, the multiple reflections will reduce. Furthermore; a full-wave optimization process carried out and an optimized orientation for sidewalls is extracted. This sidewall involves two sections of sidewall, one section absorbing the energy radiated from antenna ports and the other one absorb the energy radiated from the beam ports. With this optimized orientation, most of the incident energy radiates within a narrow field of view and the energy from the ports outside this field of view reflected onto another sidewall. The surface current distribution for one of beam ports is shown in Fig. 1 (b).

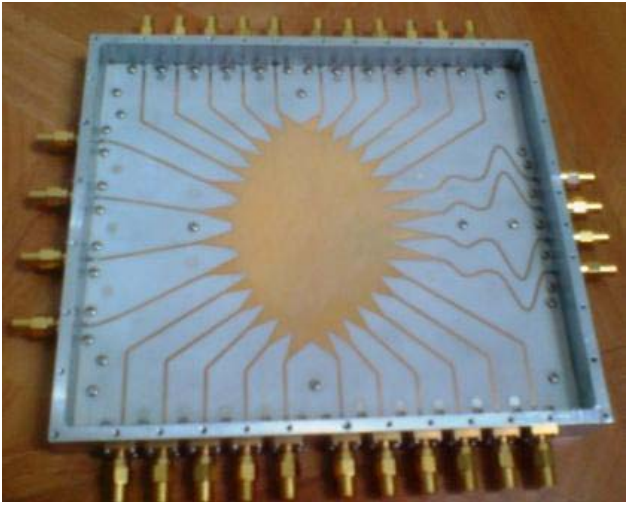

(a)

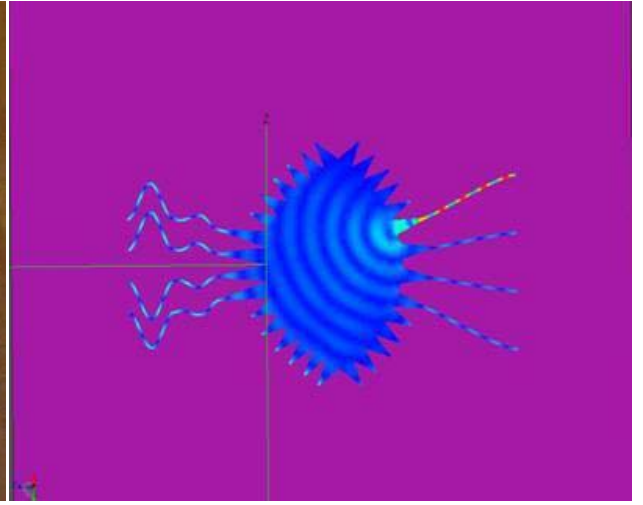

(b)

Fig. 1. (a) Prototype Lens (b) Surface current distribution for optimized

\section{Microwave lens full-wave analysis}

The proposed Rotman lens is analyzed with the method of moments (MoM) in Feko in discrete samples of frequency band and the results verified with the finite integration technique (FIT) in CST in whole frequency range from 8-26 GHz.

To capture the proposed lens general performance, we evaluate the return loss of ports, phase and amplitude coupling between each beam ports and array ports across the frequency band. 
This lens implementation has demonstrated that a match of less than $-10 \mathrm{~dB}$ is achievable form $8-26 \mathrm{GHz}$. The transmission from the beam port 1 and beam port 2 to the array ports in simulation and measurement are shown in Fig. 2 (a) Fig. 2 (f). The beam port 3 and Beam port 4 performing the same way, because the lens structure is symmetrical. The coupling from beam port to array ports demonstrated that the amplitude distribution is nearly uniform. The amplitude distribution has a low variance over the whole frequency band. Also, the maximum amplitude error for all beam ports is less than $1 \mathrm{~dB}$.

The phase shift across the aperture due to Beam port1 and beam port2 illuminations are evaluated. The simulated and measurement results show that the phase of transmission are nearly perfectly linear for transmission phase from Beam port 1 and beam port 2 to the array ports. The phase error happens when phase shift across the aperture is not linear. Similar to amplitude errors, phase error across aperture takes place for each beam port excitation. The maximum phase error in whole of frequency range is 5 degree in measurement results. Note that the maximum phase error occurs in outermost beam ports.

It is straight forward to use the standard deviation to evaluate the variation of the amplitude and phase errors due to all beam ports excitation. Fig. 2 plots the amplitude and phase error deviations for all beam ports excitation at different frequencies. Fig. 2 (e) shows that the fabricated lens maintains average amplitude error of about $0.5 \mathrm{~dB}$ for all beam ports across the entire frequencies of $8 \mathrm{GHz}$ to $26 \mathrm{GHz}$. Also, Fig. 2 (f) plots the phase error deviations for all beam ports at $8-26 \mathrm{GHz}$. It is shown that the lens has average phase error of about 2 degrees for all beam ports across the frequency range. The standard deviation of phase distribution across the outputs demonstrates the agreeable performance of prototype lens.

Whether the amplitude and phase variation are agreeable or not depends on the array's performance. By calculating the array factor, we can estimate the behavior of side lobes levels and the scanning directions. We studied the pattern performance by calculating the array factor at $8 \mathrm{GHz}$ and $18 \mathrm{GHz}$. We assume a linear array with uniform spacing of $15 \mathrm{~mm}$. The array factor gains for these frequencies are shown in Fig. 3.

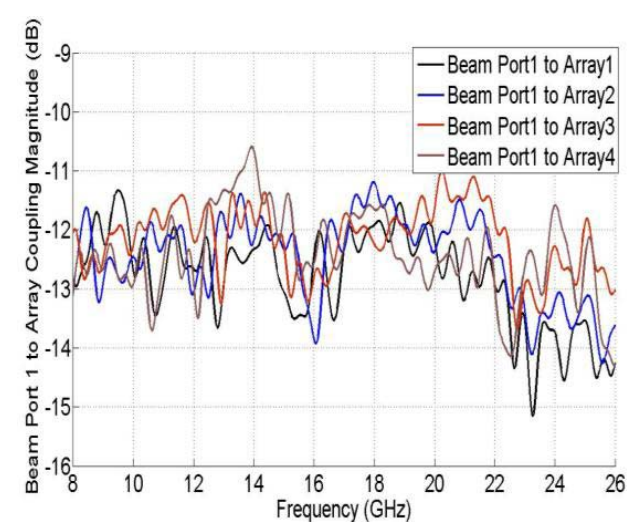

(a)

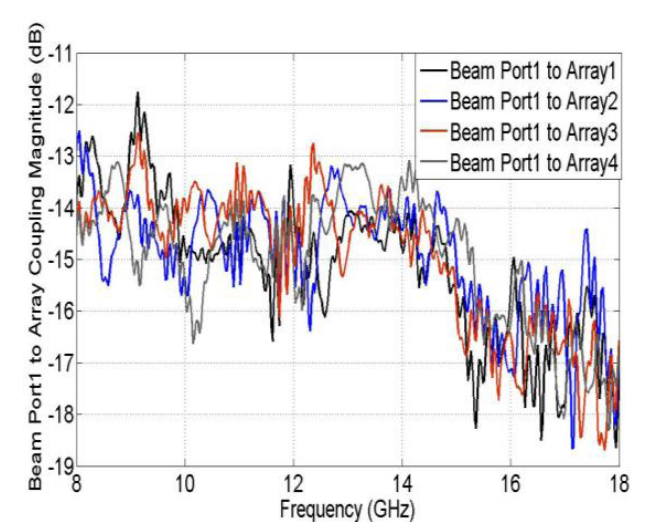

(b) 


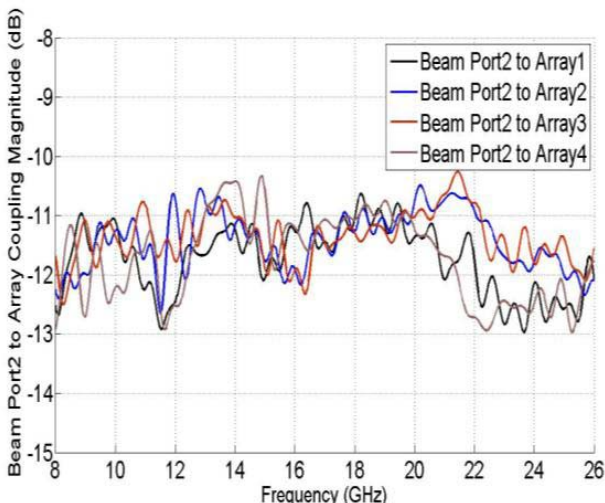

(c)

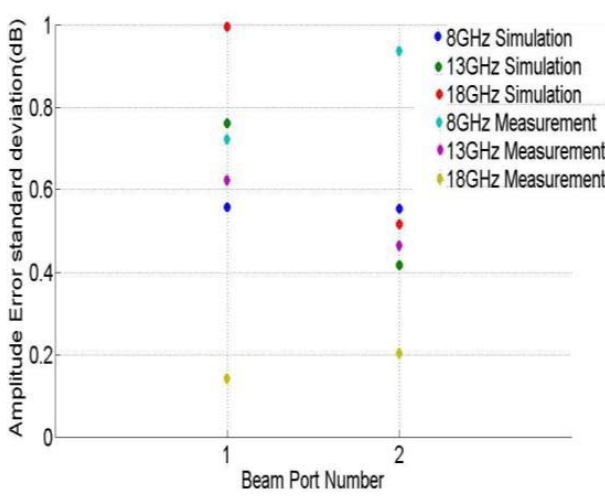

(e)

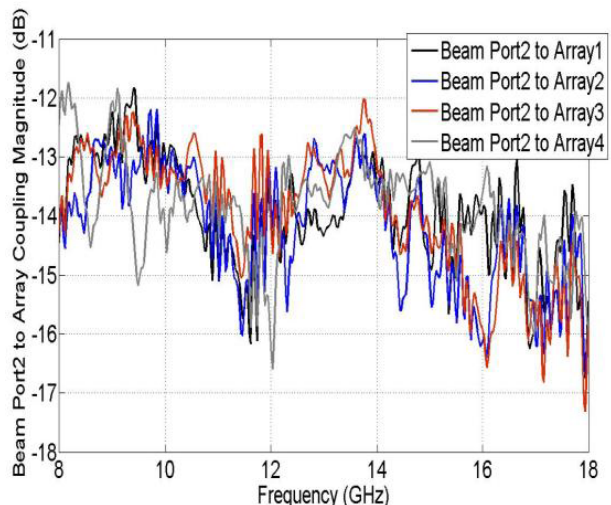

(d)

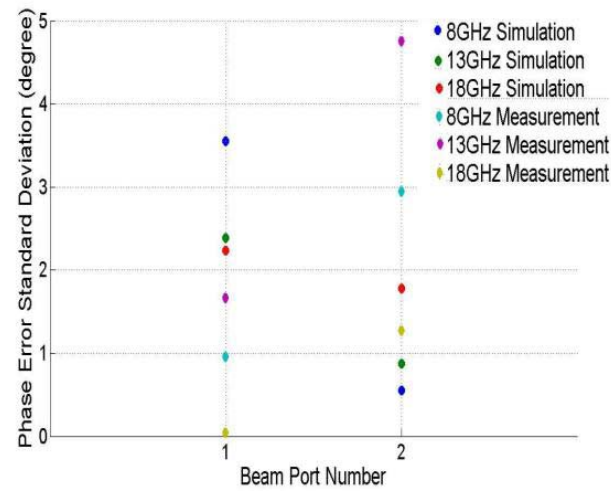

(f)

Fig. 2. (a) Beam port1 to array coupling Magnitude amplitude simulation results (b) Beam port1 to array coupling Magnitude amplitude measurement results (c) Beam port2 to array coupling Magnitude amplitude simulation results (d) Beam port2 to array coupling Magnitude amplitude measurement results (e) Amplitude Error Standard Deviation for All Beam Ports at 8-26 GHz (f) Phase Error Standard Deviation for All Beam Ports at 8-26 GHz

For single frequency operation the lens scan up to \pm 22.5 degree by four discrete steps. The side lobe levels for all frequencies in measurement are less than $-10 \mathrm{~dB}$. Comparison between Fig. 3 (a) and Fig. 3 (b) indicates that the beam width decreases and highest gain increases as the frequency increases. Note that for lower frequencies the gain difference between the measurement and simulation are less than $1 \mathrm{~dB}$. But, in higher frequencies, this difference increases that it probably due to the loss of connectors in high frequencies. For the outermost beam, gain decreases by average value of $2.6 \mathrm{~dB}$ in measurement in whole frequency band. Note that for higher frequencies the $d / \lambda$ ratio increases. Fig. 3 (b) implies that the element spacing for high frequencies is such that grating lobes radiates. The grating lobe may be suppressed somewhat by the element pattern null. But, the most 


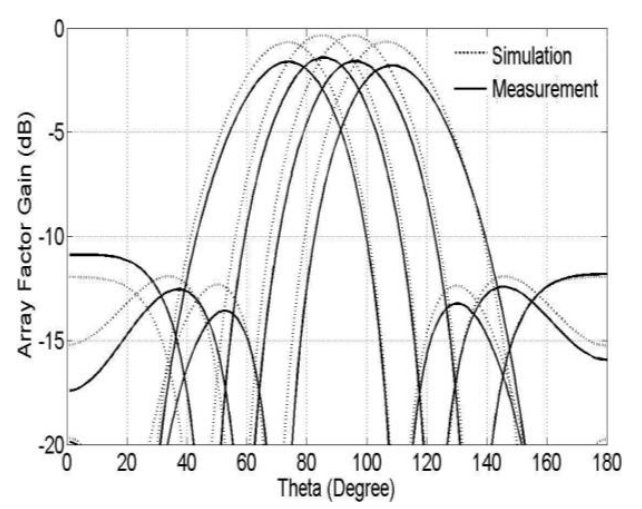

(a)

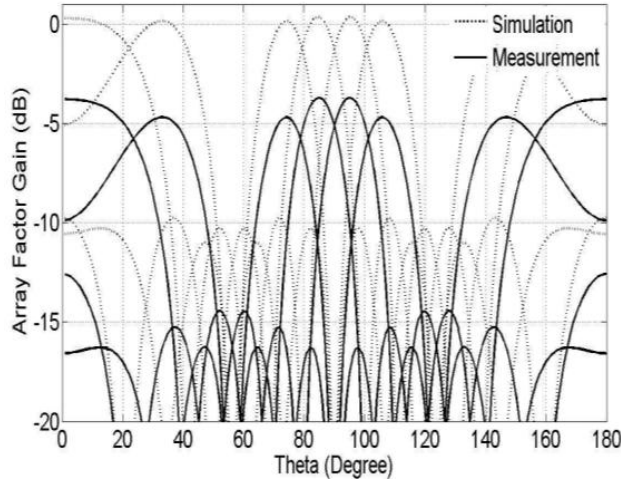

(b)

Fig. 3. (a) Array Factor Gain (8 GHZ) (b) Array Factor Gain (18 GHZ)

important thing about the lens is that all the beams have a fixed position and the lens can operate perfectly in whole frequency range from $8 \mathrm{GHz}$ to $26 \mathrm{GHz}$.

Finally, we evaluated the true time delay behavior for proposed Rotman lens. As it shows, the pointing directions of all beams remains unchanged even the frequency changes from $8 \mathrm{GHz}$ to $26 \mathrm{GHz}$. The scanning angle for all four beams between $8 \mathrm{GHz}$ to $26 \mathrm{GHz}$ deviate less than 0.65 degree from the ideal Scanning angle. So we improved the amplitude error by $33 \%$, phase error by $66 \%$ and true time delay feature of the lens by $58 \%$ in comparison with the fabricated lens in $[6,7]$.

\section{Conclusion}

Reflections from the sidewalls are the biggest limiting factor affecting the beamforming performance. An optimized orientation for dummy sidewalls is extracted using full wave optimization. Feko and CST simulation and measurements have demonstrated consistent results. The fabricated $4 \times 4$ Rotman lens has an average amplitude error of $0.5 \mathrm{~dB}$, phase errors of 2 degree, true time delay with tolerance less than 0.65 degree across $8 \mathrm{GHz}$ to $26 \mathrm{GHz}$. 